\title{
3D Hydrodynamical Simulations of a Galactic Fountain
}

\author{
M.A. de Avillez ${ }^{1}$, D.L. Berry ${ }^{1}$, and F.D. Kahn ${ }^{2}$
}

1 Departamento de Física, Universidade de Évora, R. Romão Ramalho 59, 7000 Évora, Portugal

2 Department of Physics and Astronomy, University of Manchester, Schuster Laboratory, Manchester M13 9PL, England

\begin{abstract}
Galactic fountains are thought to be responsible for the formation of the observed intermediate and high velocity clouds in the Galactic halo. Threedimensional simulations have been carried out to determine the evolution of the disk gas as it enters the fountain, cools and returns to the Galactic disk. The descending cold gas, headed by a shock, sweeps up the ascending flow, triggering the formation of an unstable layer where Rayleigh-Taylor instabilities grow, which may provoke the formation of the observed clouds.
\end{abstract}

\section{Introduction}

Gas that escapes from the Galactic disk, rises into the halo, cools and falls back, constitutes a Galactic fountain. The term "Galactic fountain" should include the outflows that result from isolated as well as clustered supernovae scattered in the Galactic disk. These outflows have been classified as Galactic fountains (Shapiro \& Field, 1976) and chimneys (Tomisaka \& Ikeuchi, 1986), and have been studied independently. Up to now three-dimensional calculations with the combined effects of such outflows have not been carried out. The fountain flow considered here results from combined processes that limit the dimensions of the chimneys and where turbulence becomes a very important factor in the formation of the observed HI clouds. Three-dimensional modelling of such a Galactic fountain will be presented here.

\section{Computational Modelling}

A three-dimensional hydrodynamical scheme described in Avillez (1997) with the adapted mesh refinement algorithm of Berger \& Collela (1989) is used to follow the evolution of the Galactic fountain. Thermal conduction is not included in the scheme but, cooling is allowed by approximating the cooling curve of Raymond et al. (1976) except in the range of temperatures $5 \times 10^{5}$ $5 \times 10^{7} \mathrm{~K}$ where Kahn's (1976) approximation is used. A section of the Galaxy centered on the Galactic plane with an area of $1 \mathrm{kpc}^{2}$ and a vertical extent from $-4 \mathrm{kpc}$ to $4 \mathrm{kpc}$ is used with the finest grid resolution being $10 \mathrm{pc}$. The 
Galactic disk is populated with randomly distributed population I stars and OB associations. Population I stars have a vertical density distribution, $\rho_{\star}$, given by (Avillez, 1997)

$$
\rho_{\star}=\rho_{\star, \mathrm{o}} \operatorname{sech}^{2}\left[\left(2 \pi G \beta_{\star} \rho_{\star}\right)^{1 / 2} z\right]
$$

where $\rho_{\star, 0}=3.0 \times 10^{-24} \mathrm{~g} \mathrm{~cm}^{-3}$ is the mass density near the Galactic plane (Allen, 1991) and the constant $\beta_{\star}=1.9 \times 10^{-13} \mathrm{~cm}^{-2} \mathrm{~s}^{2}$. This mass distribution generates a local gravitational potential with the form

$$
\Phi=-\frac{2}{\beta_{\star}} \ln \cosh \left[\left(2 \pi G \rho_{\star} \beta_{\star}\right)^{1 / 2} z\right] .
$$

Supernovae occur at a rate of three per century in the Galaxy, which corresponds to a rate of one per $1.1 \times 10^{4}$ years in the disk volume under study. The supernova remnants enter these calculations at the beginning of phase II, with initial radii depending on the density of the surrounding medium.

\section{Evolution of the Disk Gas and Halo}

The simulations have been carried out for a period of $300 \mathrm{Myr}$. The first $20 \mathrm{Myr}$ are characterized by radiative cooling excess over heating because of the small number of supernovae during this period. Thus, the gas originally located in the lower halo starts cooling and moves ballistically towards the disk, colliding there with gas falling from the opposite side of the Galactic plane. In consequence, a very thin disk of gas is formed around the Galactic plane as can be seen in Figure 1 (a) which shows HI emission at $150 \mathrm{Myr}$ after the start of calculations. The largest column density observed in the image is caused by the low scale height of the cold gas. Figure 1 (b) and (c) show the subsequent evolution of the gas at 250 , and $275 \mathrm{Myr}$, respectively. Figure 1 (b) shows the presence of low column density regions that persist for long periods. These areas are surrounded by cool shells resulting from clustered supernovae that have blown holes in the disk. In consequence hot gas escapes into the halo in a chimney-like structure. In Figure 1 (c) this region has cooled with the corresponding increase in the column density.

Colour maps of the density distribution in the galactic disk and halo obtained in these calculations are presented in Figures 1 and 2 in Kahn (1997). The density maps (Figure 1 in Kahn, 1997) show that the disk gas extends up into the halo with a stratified distribution. Low density gas located within $20 \mathrm{pc}$ of the Galactic plane, having temperatures varying between about $10^{5} \mathrm{~K}$ and $10^{6} \mathrm{~K}$, is shown in Figure 1 (in Kahn, 1997). The figure reveals the presence of cold filaments and clouds with temperatures about $10^{3} \mathrm{~K}$ distributed in the disk up to a height of $200 \mathrm{pc}$. A warm medium with temperatures of $10^{4}-10^{5} \mathrm{~K}$ is distributed up to heights of $800-900 \mathrm{pc}$. This medium is mixed with cool and cold gas that descends toward the Galactic 

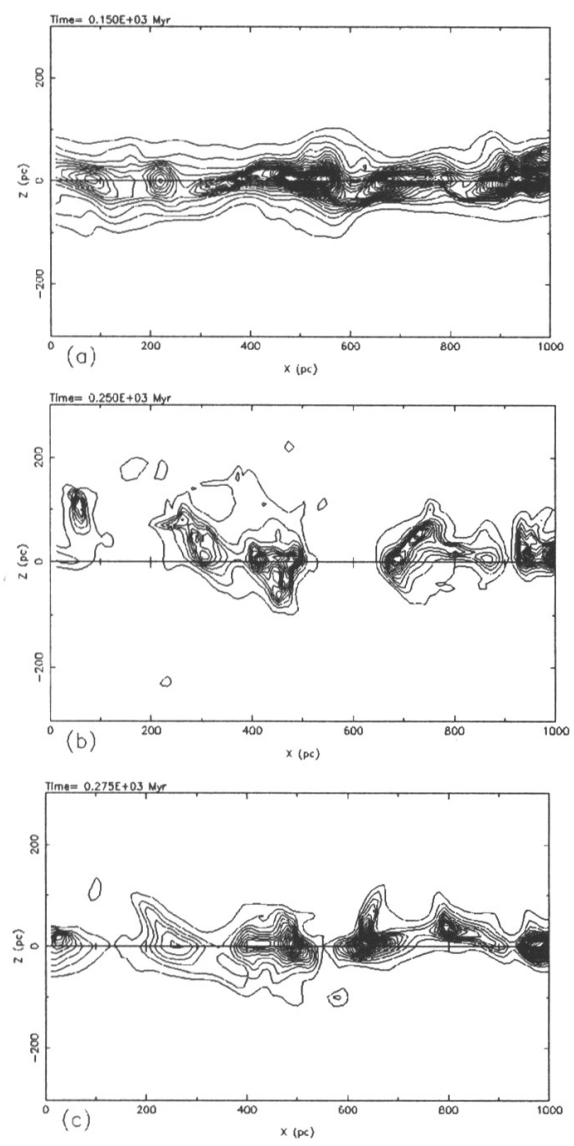

Fig. 1. Position maps of $\mathrm{HI}$ emission for the gas in a vertical section through the Galactic plane at (a) $150 \mathrm{Myr}$, (b) $250 \mathrm{Myr}$ and (c) $275 \mathrm{Myr}$ after start of calculations. The 20 equally spaced contours represent values of column density between $\log \left(N_{\mathrm{HI}}\right)=18$ and $\log \left(N_{\mathrm{HI}}\right)=19$, where $N_{\mathrm{HI}}$ is in $\mathrm{cm}^{-2}$.

disk. The turbulent warm medium supports the cool gas that sits on top of it in a kind of temporary equilibrium.

Cold HI clouds falling towards the Galactic disk are observed in the southern hemisphere (dark blue spots in Figures 2 (b-d) in Kahn, 1997), and make part of a complex with a size about 200 pc. These clouds with sizes of 20 pc typically have LSR velocities of $-190 \mathrm{~km} \mathrm{~s}^{-1}$. Most of the cold gas detected in the northern hemisphere shows LSR velocities of $-20 \mathrm{~km} \mathrm{~s}^{-1}$ to $-90 \mathrm{~km} \mathrm{~s}^{-1}$. There are two possible fates for these structures: (1) they break up into cloudlets due to Rayleigh-Taylor instabilities whenever they interact with the ascending gas from the galactic disk (Berry et al., 1997) or (2) they are disrupted by the stream of hot gas that escapes from the Galactic 
disk in chimneys (in Figure 2 (a) in Kahn, 1997; located at $x=200 \mathrm{pc}$, $z=-150 \mathrm{pc}$ ). In either case convection and turbulence occur. The disrupted gas enters the flow stream and is transported to greater heights becoming part of a diffuse medium located up to distances of $4 \mathrm{kpc}$ above the disk. Gas with temperatures smaller than $10^{4} \mathrm{~K}$ has not been detected there in these calculations.

\section{Summary}

The results reported here have a drawback, the grid resolution. Therefore, it is impossible to resolve any cool sheets with thickness of some $2 \mathrm{pc}$. Nevertheless, these results suggest that the classical picture of the Galactic fountain and chimneys must be changed. The fountain is formed by a turbulent convective flow that rises into the halo. HI clouds result from (a) cool dense shells on the outside of old supernova remnants and (b) Rayleigh-Taylor instabilities at the interface of the cooling structures that interact with the ascending flow. These structures may be related with the HI complexes observed in the halo (Wakker, 1991 and references therein). As they interact with more energetic fountain gas coming from below and specially from chimneys the clouds break up and the cool gas is swept along by the hot gas stream that eventually flows into the upper halo. Chimneys are limited to sizes no bigger than $100-120 \mathrm{pc}$ in the disk and lower halo in agreement with the observations of Normandeau et al. (1996). A stratified density distribution is found in the Galaxy, with cool and diffuse media residing in equilibrium with the layers below; this result is in agreement with observations by Kalberla et al. (1997).

Acknowledgements. This work has been sponsored by a joint grant from JNICT/ ESO (Project number PESO/C/PRO/1022/94). M.A. de A. would like to thank the LOC of this colloquium for a travel grant.

\section{References}

Allen, C.W., 1991, Astrophysical Quantities, The Athlone Press, London.

Avillez M.A., 1997, Ph.D Thesis, University of Évora.

Berry D.L., Avillez M.A. \& Kahn F.D., 1997, these proceedings.

Berger M.J. \& Colella P., 1989, J.Comput.Phys. 82, 64.

Kahn F.D., 1976, A\&A 50, 145.

Kahn F.D., 1997, these proceedings.

Kalberla P.M.W., Pietz J. \& Kerp J., 1997, these proceedings.

Normandeau M., Taylor A.R. \& Dewdney P.E., 1996, Nature 380, 687.

Raymond J.C., Cox D.P. \& Smith B.W., 1976, ApJ 204, 290.

Shapiro, P.R., Field G.B., 1976, ApJ 205, 762.

Tomisaka, K. \& Ikeuchi S., 1986, Publ. Astron. Soc. Japan 38, 697. 